\title{
Emergence and Spread of Cephalosporin-Resistant Neisseria gonorrhoeae with Mosaic penA Alleles, South Korea, 2012-2017
}

\author{
Hyukmin Lee, Young Hee Suh, Sunhwa Lee, Yong-Kyun Kim, \\ Mi-Soon Han, Hye Gyung Bae, Magnus Unemo, ${ }^{1}$ Kyungwon Lee ${ }^{1}$
}

In South Korea, surveillance of antimicrobial drug resistance in Neisseria gonorrhoeae is extremely limited. We describe the emergence and subsequent national spread of $N$. gonorrhoeae strains with mosaic penA alleles associated with decreased susceptibility and resistance to extended-spectrum cephalosporins. From 2012 through 2017, the proportion of mosaic penA alleles in gonococcal-positive nucleic acid amplification test (NAAT) specimens across South Korea increased from $1.1 \%$ to $23.9 \%$. Gonococcal strains with mosaic penA alleles emerged in the international hubs of Seoul in Gyeonggi Province and Busan in South Gyeongsang Province and subsequently spread across South Korea. Most common was mosaic penA-10.001 ( $n=572$ isolates; $94.7 \%$ ), which is associated with cefixime resistance. We also identified mosaic penA-34.001 and penA-60.001, both of which are associated with multidrug-resistant gonococcal strains and spread of cefixime and ceftriaxone resistance. Implementation of molecular resistance prediction from $N$. gonorrhoeae-positive nucleic acid amplification test specimens is imperative in South Korea and internationally.

Teisseria gonorrhoeae remains a major public health 1 threat globally (1). During the past 2 decades, $N$. gonorrhoeae strains with resistance to extended-spectrum cephalosporins (ESCs), including ceftriaxone, the last remaining option for empiric first-line gonorrhea treatment, have emerged and spread internationally, which is a serious concern worldwide (2-23). Enhanced global antimicrobial resistance (AMR) surveillance is crucial to controlling

Author affiliations: Yonsei University College of Medicine, Seoul, South Korea (H. Lee, Y.H. Suh, K. Lee); Seegene Medical Foundation, Seoul (S. Lee); Samkwang Medical Laboratories, Seoul (Y.-K. Kim); U2 Clinical Laboratories, Seoul (M.-S. Han); Green Cross Laboratories, Yongin, South Korea (H.G. Bae); World Health Organization Collaborating Centre for Gonorrhoea and other STIs, Örebro University, Örebro, Sweden (M. Unemo)

DOI: https://doi.org/10.3201/eid2503.181503 further spread of AMR strains. However, in many countries, culture-based AMR testing cannot be performed because most patients are treated empirically at primary-care facilities or hospitals where culture is unavailable and nucleic acid amplification tests (NAATs) have replaced culture for diagnosis. Consequently, molecular methods for detection of AMR determinants in N. gonorrhoeae-positive NAAT specimens are essential (21).

For the currently recommended ESCs, resistance is influenced by many different mutations in various genes that collaboratively increase the MICs of ESCs $(4,5,7,24)$. Nevertheless, the main mechanism for ESC resistance is the acquisition of a mosaic pen $A$ allele encoding a mosaic version of the ESC lethal target, penicillin-binding protein 2 (24-32). Nearly all $N$. gonorrhoeae isolates with clinical or in vitro resistance to ESCs contain a mosaic penA allele. However, isolates with decreased susceptibility or susceptibility to ESCs also can contain a mosaic pen $A$ allele; that is, different mosaic penA alleles can affect the ESC MICs differently (3-8,10-19,24-38). Accordingly, a molecular assay detecting all types of mosaic penA alleles can predict ESC resistance with a high sensitivity but lower specificity (24-31). Currently, no molecular assays for detection of mosaic penA alleles and prediction of ESC resistance are commercially available.

In South Korea, $\approx 15,000$ gonorrhea patients $(\approx 29$ patients/100,000 population) are reported to the Korean Health Insurance System (http://opendata.hira.or.kr) annually, but culture and AMR testing of $N$. gonorrhoeae are exceedingly limited $(2,3)$. ESC resistance and ESCresistant $N$. gonorrhoeae strains with the mosaic penA10.001 allele were observed in 2011 in South Korea (3). Since the early 2000s, ESC-resistant $N$. gonorrhoeae strains containing the mosaic penA-10.001, associated with cefixime resistance, have been prevalent and caused cefixime treatment failures in Japan $(4,5,7,33)$. In South

1Joint senior authors. 
Korea, molecular prediction of AMR directly from $N$. gonorrhoeae-positive NAAT samples is imperative for large-scale screening and prediction of the level and spread of ESC resistance in Korea.

We describe the emergence and subsequent national spread of $N$. gonorrhoeae strains with mosaic penA alleles in gonococcal-positive NAAT specimens across South Korea from 2012 through 2017. For molecular epidemiology, penA sequencing, $N$. gonorrhoeae multiantigen sequence typing (NG-MAST), and multilocus sequence typing (MLST) were performed on all mosaic penApositive specimens.

\section{Materials and Methods}

\section{N. gonorrhoeae-Positive NAAT Specimens}

We examined DNA extracts of NAAT specimens, mainly first-voided urine (55\%) and vaginal swab specimens $(27 \%)$, but also urethral and cervical swab specimens (18\%), positive for $N$. gonorrhoeae (n $=3,884 ; 1$ specimen per patient) by Seeplex NAATs (Seegene, http:// www.seegene.com). These DNA extracts were obtained from commercial centralized accredited laboratories (2012-2017, Seegene Medical Foundation and SamKwang Medical Laboratories; 2016-2017, U2Bio; and 2017, Green Cross Medical Foundation) in Seoul, South Korea. The DNA extracts were stored at $-70^{\circ} \mathrm{C}$ before further analysis.

All samples were collected and preserved as part of the routine diagnostics, and no patient identification data were available during the study. Therefore, ethics approval was not required.

\section{Detection of Mosaic penA Alleles}

We examined all DNA extracts for mosaic penA alleles in 2 steps: 1) screening of all types of mosaic penA alleles $(7,34)$; and 2) detection of the specific mosaic penA alleles that caused high-level resistance to ceftriaxone in the strains H041 (penA-37.001; 7) and F89 (penA-42.001; 6). For screening of all types of mosaic penA alleles, we used a previously described TaqMan probe-based real-time PCR method on a Rotor-Gene 6000 (QIAGEN, https://www. qiagen.com) (34). We then tested all DNA extracts positive for a mosaic penA allele by using a real-time PCR specific for penA-37.001 (35) and a modified hybridization probebased real-time PCR detecting penA-42.001 (36).

\section{Sequencing of Mosaic penA Alleles}

We sequenced the entire penA gene in all DNA specimens positive for a mosaic penA allele, as previously described (3,37). Among 604 mosaic penA-positive specimens, we were able to sequence 601 (99.5\%) specimens. Three $(0.5 \%)$ specimens failed to sequence due to low DNA content. The mosaic penA alleles were named by using the Neisseria gonorrhoeae Sequence Typing for Antimicrobial Resistance (NG-STAR; 38) database (https://ngstar.canada.ca/ welcome/home).

\section{Molecular Epidemiologic Characterization}

We performed NG-MAST (39) and MLST on all mosaic penA-positive specimens using the methods described on the NG-MAST (http://www.ng-mast.net) and MLST (http://pubmlst.org/neisseria) websites. We successfully typed 594 (98.3\%) of the mosaic penA-positive specimens with NG-MAST and 593 (98.2\%) specimens with MLST.

\section{Antimicrobial Agents Used for Gonorrhea Treatment}

We acquired prescription data for patients treated for gonorrhea from 2010 through 2017 in South Korea from the Korea Health Insurance Review \& Assessment Service (http://opendata.hira.or.kr). In South Korea, all clinics and hospitals participate in the national health insurance system, and reporting of prescription data and diagnosis to the Korea Health Insurance Review \& Assessment Service HIRA is mandatory. We analyzed the percentage of diagnosed gonococcal infections in patients who did not have additional sexually transmitted infections and who were treated with each specific antimicrobial drug.

\section{Statistical Analysis}

We used the Student $t$-test and a test of proportions for statistical analysis (Statistica 12.0 PL software, https://www. tibco.com). The level of significance was set at $\alpha=0.05$.

\section{Results}

\section{N. gonorrhoeae-Positive NAAT Specimens and Corresponding Patients}

The collection of DNA extracts we examined consisted of 3,884 N. gonorrhoeae-positive NAAT specimens collected across South Korea from 2012 through 2017 (Table 1). The number of specimens per year varied from 428 in 2012 to 901 in 2017 (Table 1). Data on the sex and age of patients were available for $3,422(88.1 \%)$ of specimens. The ratio of men to women was $1: 0.37$, and $71.1 \%$ of the specimens were collected from patients in their 20s (43.9\%) and $30 \mathrm{~s}(27.2 \%)$.

\section{Molecular Typing of Gonococcal Mosaic penA- Positive Specimens}

The proportion of mosaic penA alleles in the specimens increased annually, from $1.2 \%(5 / 428)$ in 2012 to $23.9 \%$ (215/901) in 2017 (Table 2). The mosaic penA-10.001, previously associated with resistance to ESCs, particularly cefixime and other oral ESCs $(4,5,33)$, was the most common mosaic penA allele $(\mathrm{n}=572 ; 94.7 \%$ of all mosaic 
Table 1. Number of Neisseria gonorrhoeae-positive nucleic acid amplification test specimens examined, by year and location, South Korea, 2012-2017

\begin{tabular}{|c|c|c|c|c|c|c|c|}
\hline \multirow[t]{2}{*}{ Location } & \multicolumn{7}{|c|}{ No. (\%) tested specimens } \\
\hline & 2012 & 2013 & 2014 & 2015 & 2016 & 2017 & Total \\
\hline Seoul metropolitan area & $192(44.9)$ & $171(32.6)$ & $185(30.6)$ & $141(23.3)$ & $338(41.2)$ & $252(28.0)$ & $1,279(32.9)$ \\
\hline Gyeonggi area, including Incheon & $71(16.6)$ & $96(18.3)$ & $100(16.6)$ & $156(25.7)$ & $191(23.3)$ & $157(17.4)$ & $771(19.9)$ \\
\hline Gangwon & $4(0.9)$ & $12(2.3)$ & $7(1.2)$ & $19(3.1)$ & $16(1.9)$ & $14(1.6)$ & $72(1.9)$ \\
\hline North Chungcheong & $6(1.4)$ & $1(0.2)$ & $16(2.6)$ & $19(3.1)$ & $25(3.0)$ & $73(8.1)$ & $140(3.6)$ \\
\hline $\begin{array}{l}\text { South Chungcheong, including } \\
\text { Daejeon }\end{array}$ & $22(5.1)$ & $21(4.0)$ & $69(11.4)$ & $62(10.2)$ & $53(6.5)$ & $103(11.4)$ & $330(8.5)$ \\
\hline North Jeolla & $2(0.5)$ & $2(0.4)$ & $13(2.2)$ & $12(2.0)$ & $24(2.9)$ & $42(4.7)$ & $95(2.4)$ \\
\hline South Jeolla, including Gwanju & $17(4.0)$ & $6(1.1)$ & $39(6.5)$ & $43(7.1)$ & $42(5.1)$ & $61(6.8)$ & $208(5.4)$ \\
\hline $\begin{array}{l}\text { North Gyeongsang, including } \\
\text { Daegu }\end{array}$ & $49(11.4)$ & $93(17.7)$ & 78 (12.9) & 93 (15.3) & $83(10.1)$ & $103(11.4)$ & $499(12.8)$ \\
\hline $\begin{array}{l}\text { South Gyeongsang, including } \\
\text { Busan and Ulsan }\end{array}$ & $65(15.2)$ & $121(23.1)$ & $97(16.1)$ & $61(10.1)$ & $46(5.6)$ & $93(10.3)$ & $483(12.4)$ \\
\hline Jeju & 0 & $1(0.2)$ & 0 & 0 & $3(0.4)$ & $3(0.3)$ & $7(0.2)$ \\
\hline Total & $428(100)$ & $524(100)$ & $604(100)$ & $606(100)$ & $821(100)$ & $901(100)$ & $3,884(100)$ \\
\hline
\end{tabular}

penA-positive specimens). The annual proportion of mosaic penA-10.001 among the mosaic penA-positive specimens varied from $60 \%$ to $100 \%$ (Table 2). Most (76.9\%) mosaic penA-10.001-positive samples belonged to MLST ST1901, but the proportion of sequence type (ST) 1901 among the mosaic penA-10.001 specimens decreased from $100 \%(5 / 5)$ in 2012 to $68 \%(143 / 210)$ in 2017 . In contrast, the proportion of other MLST STs, including ST1588 and ST7363, increased. In 2012 and 2013, NG-MAST ST2958 was the most prevalent ST, but the genetically closely related NG-MAST ST10668 was most common in 2014 and 2015. In 2016 and 2017, NG-MAST STs diversified, and ST15014 became the most common.

The mosaic penA-34.001 was found in 1-3 samples annually between 2013 and 2017, and the mosaic penA72.001 (penA-34.001+P551S) was identified in 10 samples between 2013 and 2016. These mosaic penA alleles also have been previously associated with ESC resistance internationally $(4,5,31,40)$. Of particular concern, mosaic penA60.001 , which has been found in internationally spreading ceftriaxone-resistant gonococcal strains since 2015 (12,14$16,18,19)$, was identified in a sample in 2017. This sample belonged to MLST ST1903 and NG-MAST ST16327 and was collected from a man in Seoul (Table 2).

\section{Distribution of Mosaic penA Alleles in Different Provinces}

We summarized the annual proportions of mosaic penA alleles in the 10 different provinces of South Korea from 2012 through 2017 (Figure 1). In 2012, only 5 mosaic penA-positive specimens were identified: 1 each in the capital city Seoul $(0.5 \%$ of the total number of isolates collected in this location), Gyeonggi Province surrounding Seoul (1.4\%), and North Gyeongsang Province $(2.0 \%)$ and 2 (3.1\%) in South Gyeongsang Province, which includes Busan, the second largest city in South Korea. In 2013, the proportions of mosaic penA-positive specimens in these 4 provinces increased to $4.1 \%-7.4 \%$, but no mosaic
penA-positive specimens were detected in any of the other provinces. However, in 2014, while the proportion of mosaic penA-positive specimens further increased in these 4 provinces (to $6.4 \%-16.5 \%$ ), mosaic penA-positive specimens also emerged in 4 of the 6 additional provinces (proportions of $7.2 \%-15.4 \%$ ). Mosaic penA-positive specimens were widely spread in all provinces of South Korea, except Jeju Island, in 2015 (9.7\%-34.5\%) and 2016 (3.8\%$32.0 \%$ ). In 2017, the proportion of mosaic penA-positive specimens was $>20 \%$ in all provinces, except South Jeolla Province (13.1\%), and mosaic penA-positive specimens also were found on Jeju Island (33.3\%) (Figure 1).

\section{Antimicrobial Drugs Used for Gonorrhea Treatment}

In $2010,13.1 \%$ of gonorrhea patients were treated with ceftriaxone, and $17.4 \%$ were treated with any ESC, but these proportions increased to $30.5 \%$ treated with ceftriaxone and 38.8\% treated with ESCs in 2017 (Figure 2). With the exception of ceftriaxone, cefixime is the most widely used ESC; the proportion of patients treated with cefixime increased from $2.9 \%$ in 2010 to $6.6 \%$ in 2017 . In $2010,32.8 \%$ of patients were treated with spectinomycin and $49.6 \%$ with fluoroquinolone, but these proportions decreased in 2017 to $16.0 \%$ treated with spectinomycin and $26.7 \%$ treated with fluoroquinolone (Table 3 ).

\section{Discussion}

The emergence of multidrug-resistant $N$. gonorrhoeae is compromising the treatment of gonorrhea globally. In most countries, only ESCs, azithromycin combined with ESCs, and spectinomycin remain recommended for the empiric treatment of gonorrhea $(3-5,8-10,20-23,31,32,41-44)$.

Among the ESCs, oral cefixime and the more potent injectable ceftriaxone have been recommended for the treatment of gonorrhea internationally $(4,5,10,41-44)$. However, resistance to cefixime emerged in Japan in the early 2000s, and cefixime resistance subsequently has been reported in many countries globally. Since 2010, resistance 
Table 2. Molecular typing of specimens positive for a Neisseria gonorrhoeae mosaic penA allele by year, South Korea, 2012-2017*

\begin{tabular}{|c|c|c|c|c|}
\hline Year & $\begin{array}{c}\text { No. (\%) } \\
\text { specimens with } \\
\text { mosaic penA }\end{array}$ & $\begin{array}{l}\text { Mosaic penA allele } \\
\text { (no. specimens) }\end{array}$ & $\begin{array}{c}\text { MLST ST } \\
\text { (no. specimens) }\end{array}$ & NG-MAST ST (no. specimens) \\
\hline 2012 & $5(1.2)$ & $10.001(5)$ & $1901(5)$ & $2958(5)$ \\
\hline \multirow[t]{4}{*}{2013} & $27(5.2)$ & $\begin{array}{l}10.001(17) \\
72.001(4)\end{array}$ & $1901(21)$ & $\begin{array}{c}2958(8), 10668(6), 5682(1), 6783(1), 10669(1) \\
1407(3), 436(1)\end{array}$ \\
\hline & & $10.001(1)$ & $7363(1)$ & $5308(1)$ \\
\hline & & $10.001(3)$ & ND (5) & ND (3) \\
\hline & & $34.001(2)$ & & 10670 (1), ND (1) \\
\hline \multirow[t]{5}{*}{2014} & $60(9.9)$ & $10.001(1)$ & $1588(1)$ & $6696(1)$ \\
\hline & & $10.001(54)$ & $1901(56)$ & $\begin{array}{c}10668(33), 2958(15), 11495(2), 11493(1), 11494(1), \\
11496(1), 11497(1)\end{array}$ \\
\hline & & $34.001(1)$ & & $5622(1)$ \\
\hline & & $72.001(1)$ & & $1407(1)$ \\
\hline & & $10.001(3)$ & ND (3) & ND (3) \\
\hline \multirow[t]{9}{*}{2015} & $109(18.0)$ & $10.001(2)$ & $1579(2)$ & $13376(2)$ \\
\hline & & $10.001(7)$ & $1588(7)$ & 12399 (3), 3611 (2), 6696 (1), 10679 (1) \\
\hline & & $10.001(82)$ & $1901(82)$ & $\begin{array}{c}10668(38), 2958(20), 6734(7), 11495(4), 1407(3), 6481 \\
(3), 4502(2), 6373(2), 11497(1), 13374(1), 13388(1), \\
13391(1)\end{array}$ \\
\hline & & $10.001(1)$ & $1922(1)$ & $13393(1)$ \\
\hline & & $10.001(10)$ & $7363(10)$ & $5308(8), 11562(1), 13389(1)$ \\
\hline & & $10.001(2)$ & $7371(2)$ & $13390(1), 13394(1)$ \\
\hline & & $34.001(2)$ & $7827(2)$ & $3702(2)$ \\
\hline & & $10.001(2)$ & $8137(2)$ & $13373(1), 13392(1)$ \\
\hline & & $10.001(1)$ & $10931(1)$ & $12402(1)$ \\
\hline \multirow[t]{6}{*}{2016} & $185(22.5)$ & $10.001(11)$ & $1588(11)$ & $15024(7), 15026(3), 15086(1)$ \\
\hline & & $10.001(133)$ & $1901(148)$ & $\begin{array}{l}15014(39), 15016(31), 15015(28), 7307(8), 15025(3), \\
15041(3), 15029(2), 15045(2), 11497(1), 15013(1), \\
15017(1), 15018(1), 15019(1), 15020(1), 15021(1), \\
15022(1), 15023(1), 15027(1), 15031(1), 15033(1), \\
15034 \text { (1), } 15038(1), 15042(1), 15044(1), 15048(1)\end{array}$ \\
\hline & & $27.001(2)$ & & $15015(2)$ \\
\hline & & $34.001(3)$ & & $4431(1), 15032(1), 15039(1)$ \\
\hline & & $72.001(10)$ & & $15018(7), 4431(1), 15016(1), 15030(1)$ \\
\hline & & $10.001(26)$ & $7363(26)$ & $\begin{array}{c}6910(13), 15028(4), 15037(2), 15046(2), 15012(1) \\
15040(1), 15043(1), 15049(1), 15050(1)\end{array}$ \\
\hline \multirow[t]{10}{*}{2017} & $215(23.9)$ & $10.001(40)$ & $1588(40)$ & $\begin{array}{c}15024(24), 5576(4), 3611(3), 15025(3), 15026(2), 6696 \\
(1), 7684(1), 14668(1), 16331(1)\end{array}$ \\
\hline & & $10.001(143)$ & $1901(145)$ & $\begin{array}{c}15014(33), 15016(25), 7307(17), 15015(16), 5446(10), \\
15032(3), 16324(3), 16341(3), 6734(2), 15045(2), \\
16270(2), 16325(2), 16335(2), 12402(1), 13973(1), \\
15018(1), 15019(1), 15028(1), 15029(1), 16319(1), \\
16320(1), 16321(1), 16323(1), 16328(1), 16329(1), \\
16332(1), 16333(1), 16334(1), 16336(1), 16338(1), \\
16342(1), 16343(1), 16344(1), 16346(1)\end{array}$ \\
\hline & & $34.001(2)$ & & $15032(2)$ \\
\hline & & $10.001(1)$ & $1902(1)$ & $16339(1)$ \\
\hline & & $60.001(1)$ & $1903(1)$ & $16327(1)$ \\
\hline & & $10.001(24)$ & $7363(25)$ & $\begin{array}{c}6910 \text { (13), } 16322(3), 13044(1), 13876(1), 15037(1), \\
15046(1), 16318(1), 16337(1), 16340(1)\end{array}$ \\
\hline & & $34.001(1)$ & & $11624(1)$ \\
\hline & & $10.001(1)$ & $7371(1)$ & $16330(1)$ \\
\hline & & $10.001(1)$ & $10899(1)$ & $11895(1)$ \\
\hline & & $10.001(1)$ & 11179 (1) & 16345 (1) \\
\hline
\end{tabular}

*MLST, multilocus sequence typing; ND, not determined (due to insufficient amount of DNA); NG-MAST, N. gonorrhoeae multiantigen sequence typing; ST, sequence type.

to ceftriaxone, the last remaining option for empiric firstline monotherapy, also has been described in many countries $(3-19,21,32)$. The mechanisms of resistance to these ESCs are complex and involve different mutations in the pen $A$ gene, in the promoter or coding region of $m t r R$, and in the porB $1 b$ gene. However, the main ESC-resistance determinant is evidently the acquisition of a mosaic pen $A$ allele. The mosaic penA-10.001 was shown to be strongly associated with cefixime resistance in gonococcal isolates in Japan in the early 2000s $(3-5,7,33)$, which resulted in the exclusion of cefixime from the Japanese treatment guidelines in 2006 (43). In South Korea, 1 cefixime-resistant isolate cultured in 2004 (2), 1 in 2011, and 3 in 2013 previously were reported to contain mosaic penA-10.001 

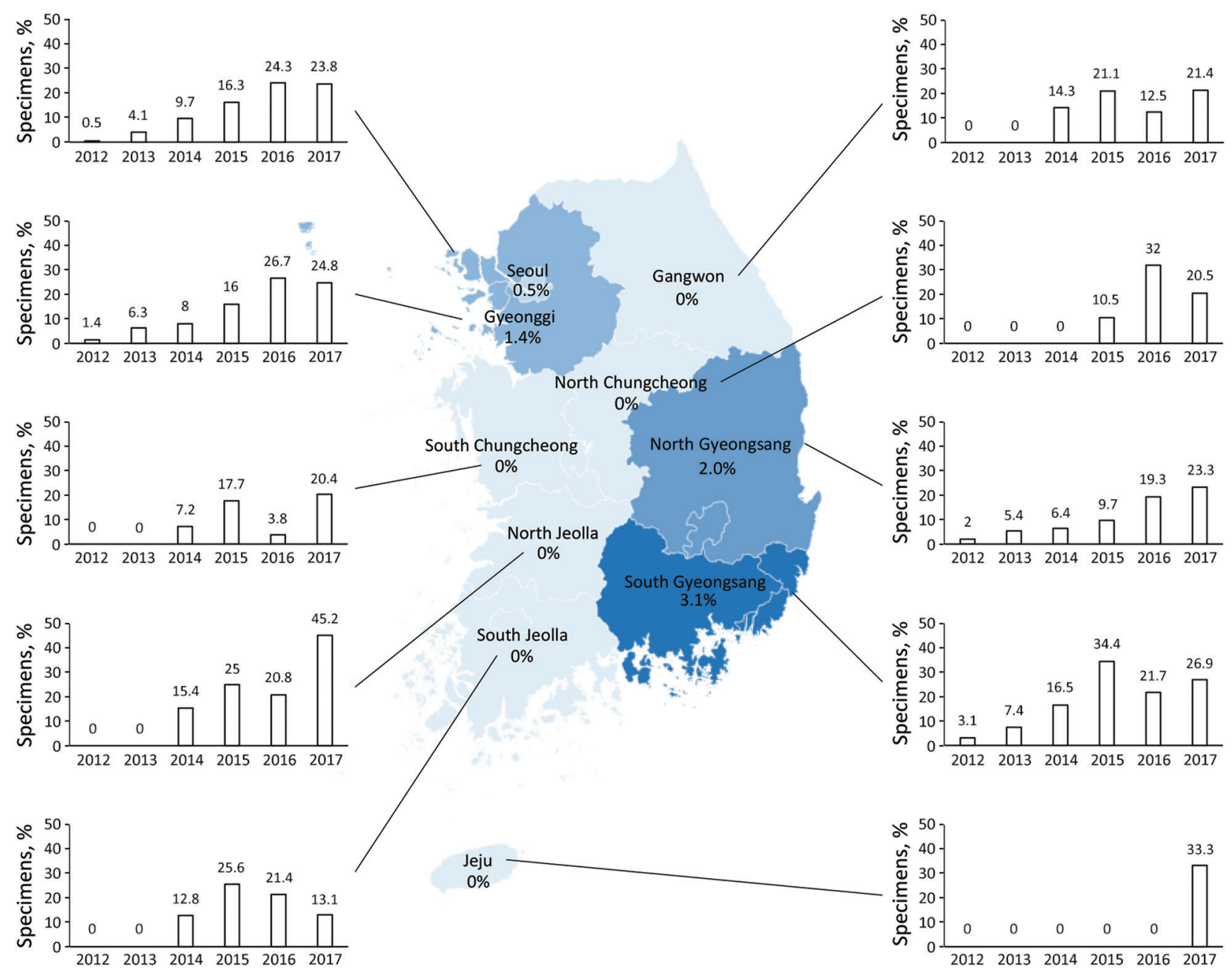

Figure 1. Distribution of specimens positive for a Neisseria gonorrhoeae mosaic penA allele, by year and province, South Korea, 2010 2017. Numbers shown in each province denote the proportion of samples positive for a mosaic penA allele in 2012. The bar graphs describe the percentage of specimens positive for a $N$. gonorrhoeae mosaic penA allele in each province and year. Seoul is the capital city of South Korea, and Gyeonggi Province contains an international airport.

(3). In our study, $1.1 \%(\mathrm{n}=5)$ of $N$. gonorrhoeae-positive NAAT specimens contained a mosaic penA allele in 2012. This proportion increased significantly in 2017 to $23.9 \%$ $(\mathrm{n}=215 ; \mathrm{p}<0.05)$. The proportion of mosaic penA-positive specimens $(\mathrm{n}=209)$ containing mosaic penA-10.001 was $100 \%(\mathrm{n}=5)$ in 2012 and $>90 \%$ during all the following years, except $2013(77.8 \% ; 21 / 27)$.

Twenty-five $(96.2 \%)$ of the NAAT specimens containing mosaic penA-10.001 in 2012 and 2013 were collected from the capital region of Seoul and surrounding Gyeonggi Province, as well as the Gyeongsang area (North and South). Seoul (population 8.8 million; 19.8\% of the population of South Korea) and the Gyeonggi area (29.6\% of the population) share 2 large international airports and an international port, which have pivotal roles in trading with foreign countries, including China and Japan.
The South Gyeongsang area (15.6\% of the population) includes Ulsan, Gyeonsangnam-do, and the second largest city in South Korea, Busan, which comprises the main port in northeast Asia, with close and frequent trading routes to Japan. In 2012 and 2013, the proportions of mosaic penA-10.001 were $2.2 \%$ in the capital area and $4.1 \%$ in the Gyeongsang area, whereas the proportion was only $0.8 \%$ in the remaining provinces. This indicates that $N$. gonorrhoeae strains with mosaic penA-10.001, associated with decreased susceptibility and resistance to ESCs, initially emerged or were imported to at least 2 areas (Seoul and Gyeongsang) of South Korea, possibly from Japan, where these strains have been prevalent for many years $(4,5,7,33)$. From 2014, the proportion of $N$. gonorrhoeae mosaic penA-10.001 in the other provinces significantly increased (from $9.1 \%$ in 2014 to $20.9 \%$ in 2017 ; $\mathrm{p}<0.05$ ); 


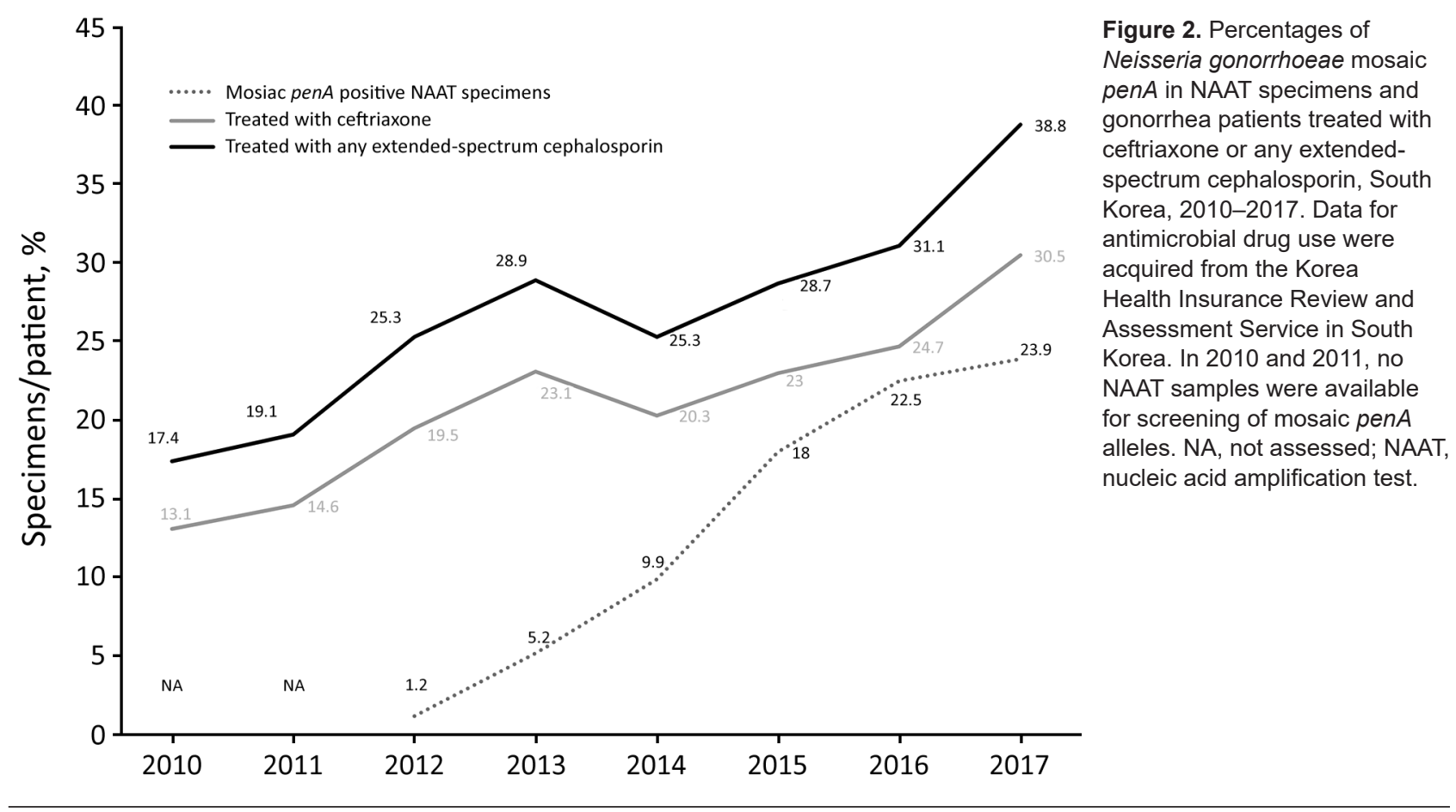

accordingly, $N$. gonorrhoeae strains with mosaic penA10.001 spread and diversified, based on NG-MAST STs, nationally in South Korea.

Gonococcal resistance to cefixime and other oral ESCs is common in South Korea, according to a smaller study (3), and the prevalent mosaic penA-10.001 we report causes decreased susceptibility and frequently resistance to cefixime and other oral ESCs $(4,5,7,33)$. With these facts in mind, oral ESCs should be abandoned from the treatment of gonorrhea in South Korea. Gonococcal strains with mosaic penA-10.001 also can cause ceftriaxone treatment failure (17). However, mosaic penA-10.001 affects the MICs of ceftriaxone less than those of cefixime in general. Thus, ceftriaxone, combined with azithromycin, should be the first-line empiric treatment in South Korea. However, N. gonorrhoeae specimens containing mosaic penA-34.001 $(\mathrm{n}=11)$ and penA-72.001 (n $=15)$ also were detected. These mosaic penA alleles have been associated with ESC resistance globally $(4,5,31,40)$ and require only a single additional penicillin binding protein 2 amino acid alteration (A501P) to develop high-level ceftriaxone resistance $(6,45)$. Of most concern, mosaic penA60.001 was found in a sample collected in Seoul in 2017 and this mosaic pen $A$ allele has been found in internationally spreading ceftriaxone-resistant gonococcal strains in Japan, Australia, Canada, Denmark, France, the United Kingdom, and Ireland since $2015(12,14-16,18,19,46)$.

The detailed reasons for the emergence and subsequent national spread of gonococcal strains with mosaic penA-10.001 in South Korea are not clear. One possibility is importation of cefixime-resistant gonococcal strains

containing the mosaic penA-10.001 from Japan, as previously mentioned. However, increased therapeutic use of ESCs, particularly oral ESCs, in South Korea cannot be excluded as contributing to the increased spread of ESCresistant gonococcal strains. In South Korea, spectinomycin, fluoroquinolones, and ESCs have been used to treat gonorrhea since the early 2000s (3). In 2002, most patients were treated with spectinomycin; only $8.4 \%$ of all patients were treated with ceftriaxone, and oral ESCs were rarely prescribed. However, $38.8 \%$ of patients were treated with ESCs (30.5\% with ceftriaxone) in 2017. Despite the high use of spectinomycin in South Korea for decades, susceptibility to spectinomycin in $N$. gonorrhoeae has remained high. No spectinomycin-resistant gonococcal strain has been reported since 1993. Nevertheless, spectinomycin has a low eradication rate for pharyngeal gonorrhea (47) and, if pharyngeal infection has not been excluded, should be used only in dual antimicrobial therapy (e.g., in combination with azithromycin). In the 2016 South Korea guideline, dual antimicrobial therapy (ceftriaxone $500 \mathrm{mg}$ or $1 \mathrm{~g}$ plus azithromycin $1 \mathrm{~g}$ ) is the recommended first-line empiric therapy for uncomplicated gonorrhea (48).

The limitations of our study include the unavailability of gonococcal isolates, MIC data, rectal or pharyngeal specimens (which are not covered by the national insurance system in South Korea), and clinical or epidemiologic information regarding patients or treatment outcome. Accordingly, phenotypic ESC resistance was not measured; instead molecular detection of mosaic penA alleles was used to reflect the ESC resistance. Nearly all gonococcal strains with ESC 
Table 3. Percentage of patients treated with each antimicrobial drug used against gonorrhea in South Korea, 2010-2017

\begin{tabular}{|c|c|c|c|c|c|c|}
\hline \multirow[b]{2}{*}{ Year } & \multicolumn{6}{|c|}{$\%$ Patients } \\
\hline & Fluoroquinolones* & Spectinomycin & Ceftriaxone & Cefixime & Other cephalosporin & Penicillin G \\
\hline 2010 & 49.6 & 32.8 & 13.1 & 2.9 & 1.4 & 0.2 \\
\hline 2011 & 51.4 & 29.2 & 14.6 & 3.7 & 0.8 & 0.2 \\
\hline 2012 & 52.3 & 22.2 & 19.5 & 4.6 & 1.2 & 0.2 \\
\hline 2013 & 47.0 & 23.9 & 23.1 & 4.6 & 1.3 & 0.2 \\
\hline 2014 & 38.8 & 22.0 & 20.3 & 3.9 & 1.1 & 0.1 \\
\hline 2015 & 39.3 & 17.7 & 23.0 & 4.5 & 1.1 & 0.1 \\
\hline 2016 & 36.7 & 17.3 & 24.7 & 5.0 & 1.4 & 0.1 \\
\hline 2017 & 26.7 & 16.0 & 30.5 & 6.6 & 1.7 & 0.1 \\
\hline
\end{tabular}

resistance contain a mosaic penA allele; however, isolates with decreased susceptibility or susceptibility to ESCs also can contain a mosaic pen $A$ allele, and different mosaic pen $A$ alleles affect the ESC MICs differently (3-8,10-19,24-38). Even so, the mosaic penA-10.001 in $94.7 \%$ of our mosaic penA allele-positive specimens is relatively strongly associated with resistance to cefixime particularly and other oral ESCs, although with a weaker association with ceftriaxone resistance. In addition, our molecular approach to identifying decreased susceptibility and resistance to ESCs can overestimate the ESC resistance. The mosaic penA allele PCR assay (34) did not include any internal control for gonococcal DNA. However, the assay has been previously evaluated on gonococcal strains and detects $1-10$ copies of a mosaic penA per PCR reaction (34) on urogenital and extragenital gonococcal-positive and gonococcal-negative NAAT samples $(49,50)$. Finally, the distribution of specimens could not be allocated by population in an ideal way and the suboptimal number of samples, especially from the Gyeonggi area, Jeju, Gangwon, South Gyeongsang, South Jeolla, and North Jeolla provinces in some years, might have underestimated the prevalence of $N$. gonorrhoeae specimens with mosaic penA allele in these provinces.

In conclusion, we describe the initial emergence and subsequent national spread of $N$. gonorrhoeae strains with mosaic penA alleles (penA-10.001 in $94.7 \%$ of specimens), associated with decreased susceptibility and resistance to ESCs, in NAAT specimens collected across South Korea from 2012 through 2017. The proportion of penA-10.001, frequently causing resistance to cefixime and other oral ESCs, increased during these years. Only a few additional mutations in penA-10.001 are required for development of high-level resistance to ceftriaxone. Furthermore, we identified mosaic penA-34.001 and penA-60.001, both associated with multidrug-resistant gonorrhea and international spread of cefixime and ceftriaxone resistance $(4,5,12,14-16,18,19,31,40)$. The mosaic penA-60.001 has been found in internationally spreading ceftriaxone-resistant gonococcal strains in Japan, Australia, Canada, Denmark, the United Kingdom, France, and Ireland since $2015(12,14-16,18,19,46)$. Nevertheless, $N$. gonorrhoeae strains with ESC resistance due to nonmo- saic pen $A$ alleles, such as penA-13.001, also are spreading in South Korea (3). Consequently, it is essential to establish a systematic, regular, and quality-assured phenotypic AMR surveillance system for $N$. gonorrhoeae in South Korea. Implementing the use of molecular methods for prediction of AMR or antimicrobial susceptibility is also crucial. These molecular methods will effectively support phenotypic AMR surveillance and enable large-scale screening of $N$. gonorrhoeae-positive NAAT specimens, which represent most of $N$. gonorrhoeae diagnostic specimens in many countries internationally.

This work was performed at the Department of Laboratory Medicine, Research Institute of Bacterial Resistance, Yonsei University College of Medicine, Seoul, South Korea. This study was supported by grants from the National Institute of Health, Korea Centers for Disease Control and Prevention, Ministry of Health and Welfare, Republic of Korea.

H.L., M.U., and K.L. designed and initiated the study; S.L., Y.-K.K., M.-S.H. and H.G.B. provided NAAT specimens; H.L., S.L., Y.-K.K., and Y.H.S. coordinated and performed all the laboratory analyses; H.L., K.L., and M.U. analyzed and interpreted all the data and wrote a first draft of the paper; and all authors read, commented on, and approved the final manuscript.

\section{About the Author}

Dr. Hyukmin Lee is an associate professor and senior researcher at the Department of Laboratory Medicine, Research Institute of Bacterial Resistance, Yonsei University College of Medicine, Seoul, South Korea. His primary research interest is clinical microbiology and molecular biology, with a special focus on gonorrhea.

This study was supported by grants from the National Institutes of Health (2016-ER4401), Korea Center for Disease Control and Prevention (2017-ER4401-01), Ministry of Health and Welfare, Republic of Korea.

\section{References}

1. Newman L, Rowley J, Vander Hoorn S, Wijesooriya NS, Unemo M, Low N, et al. Global estimates of the prevalence and incidence of four curable sexually transmitted infections in 2012 based on systematic review and global reporting. PLoS One. 2015;10:e0143304. http://dx.doi.org/10.1371/journal.pone.0143304 
2. Lee H, Hong SG, Soe Y, Yong D, Jeong SH, Lee K, et al. Trends in antimicrobial resistance of Neisseria gonorrhoeae isolated from Korean patients from 2000 to 2006. Sex Transm Dis. 2011;38:1082-6. http://dx.doi.org/10.1097/ OLQ.0b013e31822e60a4

3. Lee H, Unemo M, Kim HJ, Seo Y, Lee K, Chong Y. Emergence of decreased susceptibility and resistance to extended-spectrum cephalosporins in Neisseria gonorrhoeae in Korea. J Antimicrob Chemother. 2015;70:2536-42. http://dx.doi.org/10.1093/jac/dkv146

4. Unemo M, Shafer WM. Antimicrobial resistance in Neisseria gonorrhoeae in the 21st century: past, evolution, and future. Clin Microbiol Rev. 2014;27:587-613. http://dx.doi.org/10.1128/ CMR.00010-14

5. Unemo M, Nicholas RA. Emergence of multidrug-resistant, extensively drug-resistant and untreatable gonorrhea. Future Microbiol. 2012;7:1401-22. http://dx.doi.org/10.2217/fmb.12.117

6. Unemo M, Golparian D, Nicholas R, Ohnishi M, Gallay A, Sednaoui P. High-level cefixime- and ceftriaxone-resistant Neisseria gonorrhoeae in France: novel penA mosaic allele in a successful international clone causes treatment failure. Antimicrob Agents Chemother. 2012;56:1273-80. http://dx.doi.org/10.1128/ AAC.05760-11

7. Ohnishi M, Golparian D, Shimuta K, Saika T, Hoshina S, Iwasaku K, et al. Is Neisseria gonorrhoeae initiating a future era of untreatable gonorrhea?: detailed characterization of the first strain with highlevel resistance to ceftriaxone. Antimicrob Agents Chemother. 2011;55:3538-45. http://dx.doi.org/10.1128/AAC.00325-11

8. Bolan GA, Sparling PF, Wasserheit JN. The emerging threat of untreatable gonococcal infection. N Engl J Med. 2012;366:485-7. http://dx.doi.org/10.1056/NEJMp1112456

9. Kirkcaldy RD, Hook EW III, Soge OO, del Rio C, Kubin G, Zenilman JM, et al. Trends in Neisseria gonorrhoeae susceptibility to cephalosporins in the United States, 2006-2014. JAMA. 2015;314:1869-71. http://dx.doi.org/10.1001/jama.2015.10347

10. Unemo M. Current and future antimicrobial treatment of gonorrhoea - the rapidly evolving Neisseria gonorrhoeae continues to challenge. BMC Infect Dis. 2015;15:364. http://dx.doi.org/10.1186/s12879-015-1029-2

11. Lahra MM, Ryder N, Whiley DM. A new multidrug-resistant strain of Neisseria gonorrhoeae in Australia. N Engl J Med. 2014;371:1850-1. http://dx.doi.org/10.1056/NEJMc1408109

12. Nakayama S, Shimuta K, Furubayashi K, Kawahata T, Unemo M, Ohnishi M. New ceftriaxone- and multidrug-resistant Neisseria gonorrhoeae strain with a novel mosaic pen $A$ gene isolated in Japan. Antimicrob Agents Chemother. 2016;60:4339-41. http://dx.doi.org/10.1128/AAC.00504-16

13. Deguchi T, Yasuda M, Hatazaki K, Kameyama K, Horie K, Kato T, et al. New clinical strain of Neisseria gonorrhoeae with decreased susceptibility to ceftriaxone, Japan. Emerg Infect Dis. 2016;22:142-4. http://dx.doi.org/10.3201/eid2201.150868

14. Lahra MM, Martin I, Demczuk W, Jennison AV, Lee KI, Nakayama SI, et al. Cooperative recognition of internationally disseminated ceftriaxone-resistant Neisseria gonorrhoeae strain. Emerg Infect Dis. 2018;24:735-40. http://dx.doi.org/10.3201/eid2404.171873

15. Terkelsen D, Tolstrup J, Johnsen CH, Lund O, Larsen HK, Worning P, et al. Multidrug-resistant Neisseria gonorrhoeae infection with ceftriaxone resistance and intermediate resistance to azithromycin, Denmark, 2017. Euro Surveill. 2017;22:1273. http://dx.doi.org/10.2807/1560-7917.ES.2017.22.42.17-00659

16. Poncin T, Fouere S, Braille A, Camelena F, Agsous M, Bebear C, et al. Multidrug-resistant Neisseria gonorrhoeae failing treatment with ceftriaxone and doxycycline in France, November 2017. Euro Surveill. 2018;23. http://dx.doi.org/10.2807/1560-7917. ES.2018.23.21.1800264

17. Fifer H, Natarajan U, Jones L, Alexander S, Hughes G, Golparian D, et al. Failure of dual antimicrobial therapy in treatment of gonorrhea. N Engl J Med. 2016;374:2504-6. http://dx.doi.org/ 10.1056/NEJMc1512757

18. Eyre DW, Sanderson ND, Lord E, Regisford-Reimmer N, Chau K, Barker L, et al. Gonorrhoea treatment failure caused by a Neisseria gonorrhoeae strain with combined ceftriaxone and high-level azithromycin resistance, England, February 2018. Euro Surveill. 2018;23. http://dx.doi.org/10.2807/1560-7917. ES.2018.23.27.1800323

19. Whiley DM, Jennison A, Pearson J, Lahra MM. Genetic characterisation of Neisseria gonorrhoeae resistant to both ceftriaxone and azithromycin. Lancet Infect Dis. 2018;18:717-8. http://dx.doi.org/10.1016/S1473-3099(18)30340-2

20. Ndowa F, Lusti-Narasimhan M, Unemo M. The serious threat of multidrug-resistant and untreatable gonorrhoea: the pressing need for global action to control the spread of antimicrobial resistance, and mitigate the impact on sexual and reproductive health. Sex Transm Infect. 2012;88:317-8. http://dx.doi.org/10.1136/ sextrans-2012-050674

21. World Health Organization, Department of Reproductive Health and Research. 2012. Global action plan to control the spread and impact of antimicrobial resistance in Neisseria gonorrhoeae. Geneva: The Organization; 2012. p. 1-36.

22. Centers for Disease Control and Prevention. 2012. Cephalosporinresistant Neisseria gonorrhoeae public health response plan. Atlanta: The Centers; 2012. p. 1-43.

23. European Centre for Disease Prevention and Control. Response plan to control and manage the threat of multidrug-resistant gonorrhoea in Europe. Stockholm: The Centre; 2012. p. 1-23. http://dx.doi.org/10.2900/60053

24. Unemo M, Nicholas RA, Jerse AE, Davies C, Shafer WM. Molecular mechanisms of antibiotic resistance expressed by the pathogenic Neisseria, chapter 9. In: Davies JK, Kahler CM, editors. Pathogenic Neisseria: genomics, molecular biology and disease intervention. London: Caister Academic Press; 2014. p. 161-92.

25. Low N, Unemo M. Molecular tests for the detection of antimicrobial resistant Neisseria gonorrhoeae: when, where, and how to use? Curr Opin Infect Dis. 2016;29:45-51. http://dx.doi.org/10.1097/ QCO.0000000000000230

26. Goire N, Lahra MM, Chen M, Donovan B, Fairley CK, Guy R, et al. Molecular approaches to enhance surveillance of gonococcal antimicrobial resistance. Nat Rev Microbiol. 2014;12:223-9. http://dx.doi.org/10.1038/nrmicro3217

27. Whiley DM, Lahra MM, Unemo M. Prospects of untreatable gonorrhea and ways forward. Future Microbiol. 2015;10:313-6. http://dx.doi.org/10.2217/fmb.14.138

28. Zhao S, Duncan M, Tomberg J, Davies C, Unemo M, Nicholas RA. Genetics of chromosomally mediated intermediate resistance to ceftriaxone and cefixime in Neisseria gonorrhoeae. Antimicrob Agents Chemother. 2009;53:3744-51. http://dx.doi.org/10.1128/ AAC.00304-09

29. Tomberg J, Unemo M, Ohnishi M, Davies C, Nicholas RA. Identification of amino acids conferring high-level resistance to expanded-spectrum cephalosporins in the penA gene from Neisseria gonorrhoeae strain H041. Antimicrob Agents Chemother. 2013;57:3029-36. http://dx.doi.org/10.1128/AAC.00093-13

30. Donà V, Low N, Golparian D, Unemo M. Recent advances in the development and use of molecular tests to predict antimicrobial resistance in Neisseria gonorrhoeae. Expert Rev Mol Diagn. 2017;17:845-59. http://dx.doi.org/10.1080/14737159.2017.1360137

31. Harris SR, Cole MJ, Spiteri G, Sánchez-Busó L, Golparian D, Jacobsson S, et al.; Euro-GASP study group. Public health surveillance of multidrug-resistant clones of Neisseria gonorrhoeae in Europe: a genomic survey. Lancet Infect Dis. 2018;18:758-68. http://dx.doi.org/10.1016/S1473-3099(18)30225-1

32. Wi T, Lahra MM, Ndowa F, Bala M, Dillon JR, Ramon-Pardo P, et al. Antimicrobial resistance in Neisseria gonorrhoeae: Global 
surveillance and a call for international collaborative action. PLoS Med. 2017;14:e1002344. http://dx.doi.org/10.1371/journal. pmed. 1002344

33. Shimuta K, Watanabe Y, Nakayama S, Morita-Ishihara T, Kuroki T, Unemo M, et al. Emergence and evolution of internationally disseminated cephalosporin-resistant Neisseria gonorrhoeae clones from 1995 to 2005 in Japan. BMC Infect Dis. 2015;15:378. http://dx.doi.org/10.1186/s12879-015-1110-x

34. Ochiai S, Ishiko H, Yasuda M, Deguchi T. Rapid detection of the mosaic structure of the Neisseria gonorrhoeae penA gene, which is associated with decreased susceptibilities to oral cephalosporins. J Clin Microbiol. 2008;46:1804-10. http://dx.doi.org/10.1128/ JCM.01800-07

35. Goire N, Ohnishi M, Limnios AE, Lahra MM, Lambert SB, Nimmo GR, et al. Enhanced gonococcal antimicrobial surveillance in the era of ceftriaxone resistance: a real-time PCR assay for direct detection of the Neisseria gonorrhoeae H041 strain. J Antimicrob Chemother. 2012;67:902-5. http://dx.doi.org/10.1093/jac/dkr549

36. Goire N, Lahra MM, Ohnishi M, Hogan T, Liminios AE, Nissen MD, et al. Polymerase chain reaction-based screening for the ceftriaxone-resistant Neisseria gonorrhoeae F89 strain. Euro Surveill. 2013;18:20444. http://dx.doi.org/10.2807/1560-7917. ES2013.18.14.20444

37. Lee SG, Lee H, Jeong SH, Yong D, Chung GT, Lee YS, et al. Various pen $A$ mutations together with $m t r R$, porB and ponA mutations in Neisseria gonorrhoeae isolates with reduced susceptibility to cefixime or ceftriaxone. J Antimicrob Chemother. 2010;65:669-75. http://dx.doi.org/10.1093/jac/dkp505

38. Demczuk W, Sidhu S, Unemo M, Whiley DM, Allen VG, Dillon JR, et al. Neisseria gonorrhoeae sequence typing for antimicrobial resistance, a novel antimicrobial resistance multilocus typing scheme for tracking the global dissemination of $N$. gonorrhoeae strains. J Clin Microbiol. 2017;55:1454-68. http://dx.doi.org/ 10.1128/JCM.00100-17

39. Martin IM, Ison CA, Aanensen DM, Fenton KA, Spratt BG. Rapid sequence-based identification of gonococcal transmission clusters in a large metropolitan area. J Infect Dis. 2004;189: 1497-505. http://dx.doi.org/10.1086/383047

40. Chisholm SA, Unemo M, Quaye N, Johansson E, Cole MJ, Ison CA, et al. Molecular epidemiological typing within the European Gonococcal Antimicrobial Resistance Surveillance Programme reveals predominance of a multidrug-resistant clone. Euro Surveill. 2013;18:ii-20358.

41. Bignell C, Unemo M, European STI Guidelines Editorial Board. 2012 European guideline on the diagnosis and treatment of gonorrhoea in adults. Int J STD AIDS. 2013;24:85-92. http://dx.doi.org/10.1177/0956462412472837
42. Workowski KA, Bolan GA; Centers for Disease Control and Prevention. Sexually transmitted diseases treatment guidelines, 2015. MMWR Recomm Rep. 2015;64(RR-03):1-137.

43. Japanese Society of Sexually Transmitted Infection. Gonococcal infection. Sexually transmitted infections, diagnosis and treatment guidelines 2011. Jpn J Sex Transm Dis. 2011; 22(Suppl 1):52-9.

44. World Health Organization, Department of Reproductive Health and Research. WHO guidelines for the treatment of Neisseria gonorrhoeae. Geneva: The Organization; 2016. p. 1-64.

45. Cámara J, Serra J, Ayats J, Bastida T, Carnicer-Pont D, Andreu A, et al. Molecular characterization of two high-level ceftriaxoneresistant Neisseria gonorrhoeae isolates detected in Catalonia, Spain. J Antimicrob Chemother. 2012;67:1858-60. http://dx.doi.org/10.1093/jac/dks162

46. Golparian D, Rose L, Lynam A, Mohamed A, Bercot B, Ohnishi M, et al. Multidrug-resistant Neisseria gonorrhoeae isolate, belonging to the internationally spreading Japanese FC428 clone, with ceftriaxone resistance and intermediate resistance to azithromycin, Ireland, August 2018. Euro Surveill. 2018;23:1800617.

47. Moran JS. Treating uncomplicated Neisseria gonorrhoeae infections: is the anatomic site of infection important? Sex Transm Dis. 1995;22:39-47. http://dx.doi.org/10.1097/ 00007435-199501000-00007

48. Korean Association of Urogenital Tract Infection and Inflammation and Korea Centers for Disease Control and Prevention. Korean guidelines for sexually transmitted infections. Osong: Korea Centers for Disease Control and Prevention; 2016.

49. Goire N, Freeman K, Lambert SB, Nimmo GR, Limnios AE, Lahra MM, et al. The influence of target population on nonculture-based detection of markers of Neisseria gonorrhoeae antimicrobial resistance. Sex Health. 2012;9:422-9. http://dx.doi.org/ 10.1071/SH12026

50. Gose S, Nguyen D, Lowenberg D, Samuel M, Bauer H, Pandori M. Neisseria gonorrhoeae and extended-spectrum cephalosporins in California: surveillance and molecular detection of mosaic penA. BMC Infect Dis. 2013;13:570. http://dx.doi.org/10.1186/ 1471-2334-13-570

Address for correspondence: Kyungwon Lee, Yonsei University College of Medicine, Department of Laboratory Medicine and Research Institute of Bacterial Resistance, Seoul 129-752, Korea; email: leekcp@yuhs.ac; Magnus Unemo, WHO Collaborating Centre for Gonorrhoea and other STIs, Department of Laboratory Medicine, Microbiology, Örebro University Hospital, SE-701 85 Örebro, Sweden; email: magnus.unemo@regionorebrolan.se 